[Article]

\title{
2,5-二氟苯酚分子之旋转异构物的质量分辨國值电离光谱
}

\author{
吴沛颖 ${ }^{1,2}$ 曾圣渊 ${ }^{1}$ 蔡青妘 ${ }^{1,3}$ 曾文碧 ${ }^{1,3,{ }^{*}}$ \\ ( ${ }^{1}$ 台湾中央研究院原子与分子科学研究所, 生化分子质谱与光谱学实验室, 台北 10617; \\ 2 台湾大学化学系, 台北 10617; ${ }^{3}$ 台湾师范大学化学系, 台北 11677)
}

\begin{abstract}
摘要: 利用共振双光子电离(R2PI)技术和质量分辨阈值电离(MATI)技术来研究 2,5-二氟苯酚分子。实验所测 得的顺式、反式 2,5-二氟苯酚分子电子激发能 $E_{1}$ 分别为 36448 和 $36743 \mathrm{~cm}^{-1}$, 绝热电离能分别为 71164 和 $71476 \mathrm{~cm}^{-1}$ 。这两个顺反转动同素异构分子在电子激发 $S_{1}$ 态与离子 $D_{0}$ 态活性振动主要是由于面内环变形和 与取代基相关的弯曲振动。分析 2,5 -二氟苯酚分子的振动光谱、 $D_{0}$ 态离子光谱以及理论计算均表明这两个转 动异构体在 $D_{0}$ 态的几何构型与 $S_{1}$ 态的中性几何构型相当相似。
\end{abstract}

关键词：共振双光子电离技术; 阈值电离; 振动光谱; 离子态光谱; 2,5 -二氟苯酚

中图分类号: 0643

\section{Rotamers of 2,5-Difluorophenol Studied Using Mass-Analyzed Threshold Ionization Spectroscopy}

\author{
WU Pei-Ying ${ }^{1,2} \quad$ TZENG Sheng-Yuan ${ }^{1} \quad$ TSAI Ching-Yun ${ }^{1,3} \quad$ TZENG Wen-Bih ${ }^{1,3, *}$ \\ ('Laboratory for Mass Spectrometric and Spectroscopic Studies of Biomolecules, Institute of Atomic and Molecular Sciences, \\ Academia Sinica, Taipei 10617; ${ }^{2}$ Department of Chemistry, National Taiwan University, Taipei 10617; \\ ${ }^{3}$ Department of Chemistry, National Taiwan Normal University, Taipei 11677)
}

\begin{abstract}
We applied resonant two-photon ionization (R2PI) and mass-analyzed threshold ionization (MATI) techniques to record the vibronic and cationic spectra of 2,5-difluorophenol. The distinct bands at 36448 and $36743 \mathrm{~cm}^{-1}$ were confirmed as the origins of the $S_{1} \leftarrow S_{0}$ electronic transition of the cis and trans rotamers, respectively. The corresponding adiabatic ionization energies were found to be 71164 and $71476 \mathrm{~cm}^{-1}$ for these two rotameric species. The observed spectral features mainly result from the in-plane ring deformation and substituent-sensitive bending vibrations. Spectral analysis suggests that the molecular geometry and vibrational coordinates of the cation in the $D_{0}$ state resemble those of the neutral species in the $S_{1}$ state for both cis and trans rotamers.
\end{abstract}

Key Words: Resonant two-photon ionization technique; Threshold ionization; Vibronic spectrum; Cation spectrum; 2,5-Difluorophenol

\section{Introduction}

Multiply substituted benzenes may have various types of structural isomers, resulting from different locations and configurations of the substituents. The nature as well as the substituent-ring and substituent-substituent interactions cause the structural isomers to have different molecular properties. One common type of structural isomers is positional isomer. For example, Silva and Ferreira ${ }^{1}$ performed combustion calorimetric studies to determine the standard molar enthalpies of formation of the six positional isomers of difluorophenol. IR, Raman, and laser-

Received: November 30, 2015; Revised: January 28, 2016; Published on Web: January 29, 2016.

${ }^{*}$ Corresponding author. Email: wbt@sinica.edu.tw Tel: +886-2-23668236; Fax: +886-2-23620200.

The project was supported by the Ministry of Science and Technology of the Republic of China (MOST-103-2113-M-001-009, MOST-104-2113-M001-014) .

国家科技部(MOST-103-2113-M-001-009, MOST-104-2113-M-001-014)资助项目

(c) Editorial office of Acta Physico-Chimica Sinica 
induced fluorescence (LIF) spectroscopies are important tools to investigate the molecular geometry, vibration, and other properties of positional isomers in the ground $S_{0}$ and electronically excited $S_{1}$ states $^{2-7}$. Another type of structural isomers is rotational isomer (rotamer) or called configurational isomer. Molecular rotamers can be interconverted by rotation about a chemical bond and coexist in a chemical sample ${ }^{8}$. It has been reported that rotamers can involve in biological processes ${ }^{9,10}$. Knowledge about molecular rotamers can help people to have more insights into some biological phenomena. Concerning positional isomers of difluorophenol, Chakrabarti and Jaman ${ }^{11}$ applied radiofrequency-microwave double resonance and conventional microwave spectroscopy to study 2,4-difluorophenol in the gas phase. They proposed that the $c i s$ and trans rotamers coexist in the sample. However, the cis form is more abundant than the trans form. Shivatare and Tzeng ${ }^{12}$ studied this molecule by using the one-color and two-color resonant two-photon ionization (1C-R2PI, 2C-R2PI), and mass-analyzed threshold ionization (MATI) techniques. They found that only the cis form involved in the two-photon photoexcitation and ionization processes. Tsai and Tzeng ${ }^{13}$ recorded the vibronic and cation spectra and reported the electronic excitation and ionization energies as well as the active vibrations of the cis and trans rotamers of 3,4-difluorophenol in the electronically excited $S_{1}$ and cationic ground $D_{0}$ states. In the present spectroscopic study, we choose 2,5-difluorophenol to learn the effects of substituent-ring and substituent - substituent interactions on molecular geometry, transition energy, and vibrational frequency. Although no optical spectrum of 2,5-difluorophenol has been reported, the existing experimental data of 2-fluorophenol ${ }^{14-16}, 2$,4-difluorophenol ${ }^{11,12}$, 3-fluorophenol ${ }^{15,17,18}$, 4-fluorophenol ${ }^{15,19}, 3$,4-difluorophenol ${ }^{13}$, and 2,5-difluoroaniline ${ }^{20}$ are useful for spectral assignment and interpretation.

Some molecular properties of specific rotamers in the $S_{0}$ and $S_{1}$ states can be obtained by combining supersonic jet-cooled expansion and $\mathrm{LIF}^{8,21}$, resonance-enhanced multiphoton ionization (REMPI $^{22,23}$, spectral hole-burning ${ }^{24,25}$ spectroscopic techniques, and theoretical calculations. However, these methods cannot give information about the ionic properties of each selected rotameric species. One may couple the resonant two photon excitation scheme $^{26}$ with either zero-kinetic energy (ZEKE) photoelectron ${ }^{27-29}$ or MATI spectroscopy ${ }^{30-33}$ to record vibrationally resolved cation spectra of the selected rotamers. Because the MATI technique provides mass information, it is suitable for spectroscopic studies of selected species in molecular systems which may have impurities ${ }^{34}$.

In this paper, we report the vibronic, photoionization efficiency (PIE), and cation spectra of 2,5-difluorophenol, recorded by the 1C-R2PI, 2C-R2PI, and MATI techniques. In particular, the MATI spectra were recorded by ionizing via different vibronic intermediate states of the selected rotamers. This allowed us to detect more active cation vibrations and to investigate whether the molecular geometry changes upon $D_{0} \leftarrow S_{1}$ transition. These new experimental data provide information about the precise excitation energies of the $S_{1} \leftarrow S_{0}$ transition $\left(E_{1}\right)$, adiabatic ionization ener- gies (IE), and the active vibrations in the $S_{1}$ and $D_{0}$ states.

\section{Experimental and computational methods}

\subsection{Experimental method}

All experiments were performed by using a photoionization time-of-flight mass spectrometer described previously ${ }^{35}$. The solid 2,5-difluoropnenol (97\%, Aldrich) sample was heated to about $77^{\circ} \mathrm{C}$ to obtain sufficient vapor pressure. The vapors were seeded into 2-3 Pa of helium and expanded into the vacuum through a pulsed valve with a $0.15 \mathrm{~mm}$ diameter orifice. The molecular beam was collimated by a skimmer located $15 \mathrm{~mm}$ downstream from the nozzle orifice. The two-color resonant two-photon excitation process was achieved by utilizing two independent tunable UV lasers controlled by a delay/pulse generator. The excitation (pump) source is a Nd:YAG pumped dye laser (Quanta-Ray PRO190-10/Lambda- Physik, Scanmate UV with BBO- III crystal; Coumarin-540A and Fluorocence-548dyes). The output visible radiation is frequency doubled to produce UV radiation. The ionization (probe) UV laser (Lambda-Physik, Scanmate UV with BBO-III crystal; Rhodamine-590 and Rhodamine-610 dyes) is pumped by a frequency-doubled Nd-YAG laser (Quanta-Ray LAB150). The wavelengths of both lasers were calibrated by using a laser wavelength meter (Coherent, WaveMaster). These two counter-propagating laser beams were focused and intersected perpendicularly with the molecular beam at $50 \mathrm{~mm}$ downstream from the nozzle orifice.

In the MATI experiments, both prompt ions and Rydberg neutrals were formed simultaneously in the laser-molecular beam interaction zone. A pulsed electric field of $-1 \mathrm{~V} \cdot \mathrm{cm}^{-1}$ was applied to the laser-molecular beam interaction region to guide the prompt ions toward the opposite direction of the flight tube. So that the prompt ions were not detected by the particle detector. It follows that the Rydberg neutrals kept moving with molecular beam velocity of about $1500 \mathrm{~m} \cdot \mathrm{s}^{-1}$ towards the direction of the flight tube. After a time delay of about $11.8 \mu \mathrm{s}$, a second pulsed electric field of $+200 \mathrm{~V} \cdot \mathrm{cm}^{-1}$ was applied to field-ionize these Rydberg neutrals in the field-ionization region. The newly formed threshold ions were accelerated and passed through a $1.0 \mathrm{~m}$ field-free region flight tube before being detected by a microchannel plate (MCP) particle detector. The obtained optical spectra were normalized to the laser power in order to avoid spurious signals due to shot-toshot laser fluctuation.

\subsection{Computational method}

All the calculations were performed by using the Gaussian 09 program package $\mathrm{e}^{36}$. The results predict the optimized structures, energies, vibrational frequencies, and many other molecular properties in the $S_{0}, S_{1}$, and $D_{0}$ states. These calculations give a total spin $\left\langle S^{2}\right\rangle$ of about 0.75 for the cation. The computed vibrational frequencies are multiplied by a suitable factor (specified in the tables) and used to assign the bands in the spectra. The IE was deduced from the difference in the zero-point energy (ZPE) levels of the cation in the $D_{0}$ state and the corresponding neutral species in the $S_{0}$ state. 


\section{Results}

\subsection{Vibronic spectrum of 2,5-difluorophenol}

Information about the $E_{1}$ of 2,5-difluorophenol is not available in literature. With the reported $E_{1}$ and IEs of 2-fluorophenol ${ }^{14-16}$ and 3-fluorophenol ${ }^{15,17,18}$ and additivity rule on the transition energy $^{13,20}$, we expect that the 1C-R2PI technique can be used to obtain the vibronic spectrum of 2,5-difluorophenol. As seen in Fig.1, the distinct features at 36448 and $36743 \mathrm{~cm}^{-1}$ are identified as the band origins of cis- and trans-2,5-difluorophenol, respectively. Table 1 lists the observed vibronic bands along with the excitation energy, relative intensity, energy shift with respect to the band origin, and possible assignment. The spectral assignment was accomplished by comparing these experimental data of 2,5difluorophenol with those of 2-fluorophenol ${ }^{14-16}, 3$-fluorophenol $^{15,17,18}, 2,4$-difluorophenol ${ }^{11,12}, 3,4$-difluorophenol ${ }^{13}$, and 2,5difluoroaniline ${ }^{20}$ as well as the predicted vibrational frequencies from our time-dependent Becke three-parameter with the LeeYang-Parr functional (TD-B3LYP) calculations. We follow the GaussView $^{37}$ procedure to identify all normal vibrations. According to Varsanyi and Szoke ${ }^{38,39}, 2,5$-difluorophenol is classified as a 1,2,5-tri-"light"-substituted benzene derivative. The numbering system of the normal vibrations is given in the corresponding nomenclature which is derived from Wilson's notation of the benzene modes ${ }^{40}$.

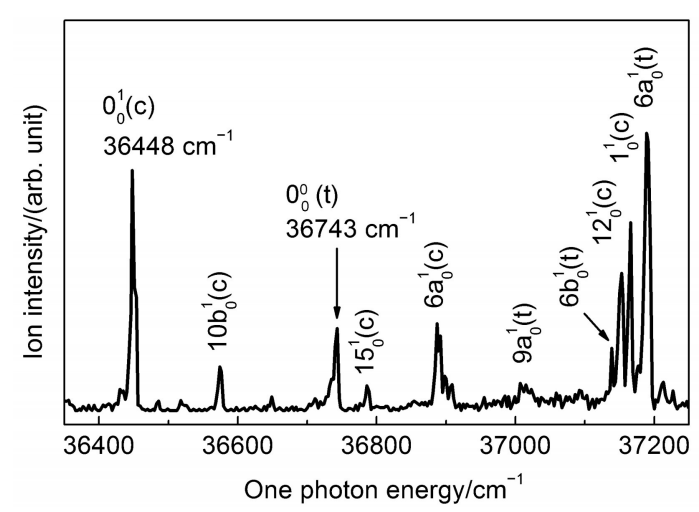

Fig.1 Vibronic spectrum of 2,5-difluorophenol obtained by the 1C-R2PI technique

The band origins of the cis and trans rotamers appear at 36448 and $36743 \mathrm{~cm}^{-1}$, respectively. $(\mathrm{c})=$ cis rotamer; $(\mathrm{t})=$ trans rotamer
The spectral features in Fig.1 mainly result from vibronic transitions related to the in-plane ring deformation and substituentsensitive bending vibrations. Modes 6a, 12, and 1 of cis-2,5difluorophenol in the $S_{1}$ state have frequencies of 439,705 , and $718 \mathrm{~cm}^{-1}$, whereas modes $6 \mathrm{~b}$ and $6 \mathrm{a}$ of trans-2,5-difluorophenol have 396 and $446 \mathrm{~cm}^{-1}$, respectively. The weak bands at 126 and $338 \mathrm{~cm}^{-1}$ are assigned to vibronic transitions $10 \mathrm{~b}_{0}^{1}$, and $15_{0}^{1}$ of cis2,5-difluorophenol, respectively. Mode 10b mainly involves outof-plane $\mathrm{C}-\mathrm{OH}$ bending motion, whereas mode 15 corresponds to in-plane $\mathrm{C}-\mathrm{F}$ bending vibrations. The band at $37007 \mathrm{~cm}^{-1}$ is tentatively assigned to vibronic transition $9 \mathrm{a}^{1}{ }_{0}$, which mainly involves in-plane $\mathrm{C}-\mathrm{OH}$ bending vibration.

\subsection{Cation spectra of cis- and trans-2,5- difluorophenol}

We applied the 2C-R2PI technique to record the PIE spectra of cis- and trans-2,5-difluorophenol by ionizing via their respective $S_{1} 0^{\circ}$ levels. Because this method detects the total ion current, it leads to an abrupt change near the ionization limit in the photoionization curve, as seen in Fig.2. The abruptly rising step indicates the IE of the selected molecular species with an uncertainty of about $10 \mathrm{~cm}^{-1}$.

Because the MATI experiment detects threshold ions, it yields a sharp peak at the ionization threshold and gives a more precise adiabatic IE. In addition, it reveals the active vibrations of the cisand trans-2,5-difluorophenol cations, as seen in Fig.3. Analysis of the $0^{+}$bands with consideration of the uncertainty in the laser photon energy, spectral width, and Stark effect, yields the adiabatic IEs of cis- and trans-2,5-difluorophenol to be (71164 \pm 5$)$ $\mathrm{cm}^{-1}((8.8232 \pm 0.0006) \mathrm{eV})$ and $(71476 \pm 5) \mathrm{cm}^{-1}((8.8619 \pm$ $0.0006) \mathrm{eV})$, respectively. It is evident that the MATI experiment gives more precise IEs than those estimated by the PIE experiment, mentioned previously.

The distinct MATI bands in Fig.3(a) shifted from the ionization threshold (denoted by $0^{+}$) by $434,501,725$, and $758 \mathrm{~cm}^{-1}$ result from the in-plane ring deformation vibrations $6 \mathrm{~b}^{1}, 6 \mathrm{a}^{1}, 12^{1}$, and $1^{1}$ of the cis-2,5-difluorophenol cation in the $D_{0}$ state, respectively, as listed in Table 2. These vibrations are also observed for the trans-2,5-difluorophenol cation. Fig.4(a-e) shows the MATI spectra of cis-2,5-difluorophenol, recorded by ionizing via the $0^{\circ}$ $\left(36448 \mathrm{~cm}^{-1}\right), 10 \mathrm{~b}^{1}\left(0^{0}+126 \mathrm{~cm}^{-1}\right), 6 \mathrm{a}^{1}\left(0^{0}+439 \mathrm{~cm}^{-1}\right), 12^{1}\left(0^{0}+\right.$

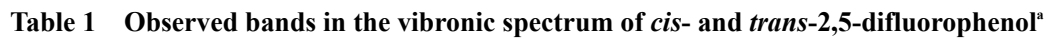

\begin{tabular}{|c|c|c|c|c|c|}
\hline & Energy $/ \mathrm{cm}^{-1}$ & Relativeintensity & Shift $/ \mathrm{cm}^{-1}$ & Cal. $\operatorname{shift}^{\mathrm{t}} / \mathrm{cm}^{-1}$ & Assignment and approximate description \\
\hline \multirow[t]{6}{*}{ cis } & 36448 & 87 & & & $0_{0}^{0}$, band origin \\
\hline & 36574 & 16 & 126 & 130 & $10 \mathrm{~b}_{0}^{1}, \gamma(\mathrm{C}-\mathrm{OH}), \gamma(\mathrm{C}-\mathrm{F})$ \\
\hline & 36786 & 9 & 338 & 323 & $15_{0}^{1}, \beta(\mathrm{C}-\mathrm{F})$ \\
\hline & 36887 & 27 & 439 & 448 & $6 \mathrm{a}_{0}^{1}, \beta(\mathrm{CCC})$ \\
\hline & 37153 & 50 & 705 & 669 & $12_{0}^{1}, \beta(\mathrm{CCC})$ \\
\hline & 37166 & 68 & 718 & 722 & $1_{0}^{1}$, breathing \\
\hline \multirow[t]{4}{*}{ trans } & 36743 & 30 & & & $0_{0}^{0}$, band origin \\
\hline & 37007 & 10 & 264 & 288 & $9 \mathrm{a}_{0}^{1}, \beta(\mathrm{C}-\mathrm{OH}), \beta(\mathrm{C}-\mathrm{F})$ \\
\hline & 37139 & 22 & 396 & 387 & $6 b_{0}^{1}, \beta(C C C)$ \\
\hline & 37187 & 100 & 446 & 439 & $6 \mathrm{a}_{0}^{1}, \beta(\mathrm{CCC})$ \\
\hline
\end{tabular}

${ }^{a}$ The predicted values are obtained from the TD-B3LYP/6-311++G( $\left.d, p\right)$ calculations, scaled by 0.97 . ${ }^{\mathrm{b}} \beta$, in-plane bending; $\gamma$, out-of-plane bending 


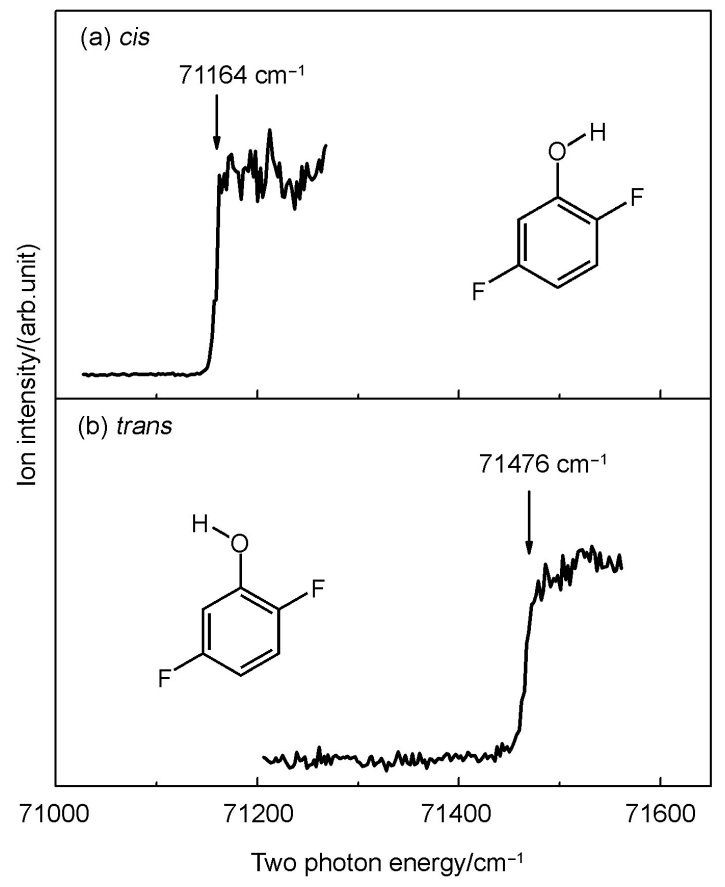

Fig.2 PIE curves of (a) cis-2,5-difluorophenol and (b) trans-2,5difluorophenol, recorded by ionizing via their respective $S_{1} 0^{\circ}$ states at 36448 and $36743 \mathrm{~cm}^{-1}$

$\left.705 \mathrm{~cm}^{-1}\right), 1^{1}\left(0^{0}+718 \mathrm{~cm}^{-1}\right)$ levels in the $S_{1}$ state. All the observed MATI bands are listed in Table 2. When the $S_{1} 10 \mathrm{~b}^{1}$ state is used as the intermediate level, a few MATI bands are observed. As stated previously, this mode mainly corresponds to the out-of-plane $\mathrm{C}-$ $\mathrm{OH}$ bending motion with some contribution of the out-of-plane substituent-sensitive $\mathrm{C}-\mathrm{F}$ bending motion. It is expected that

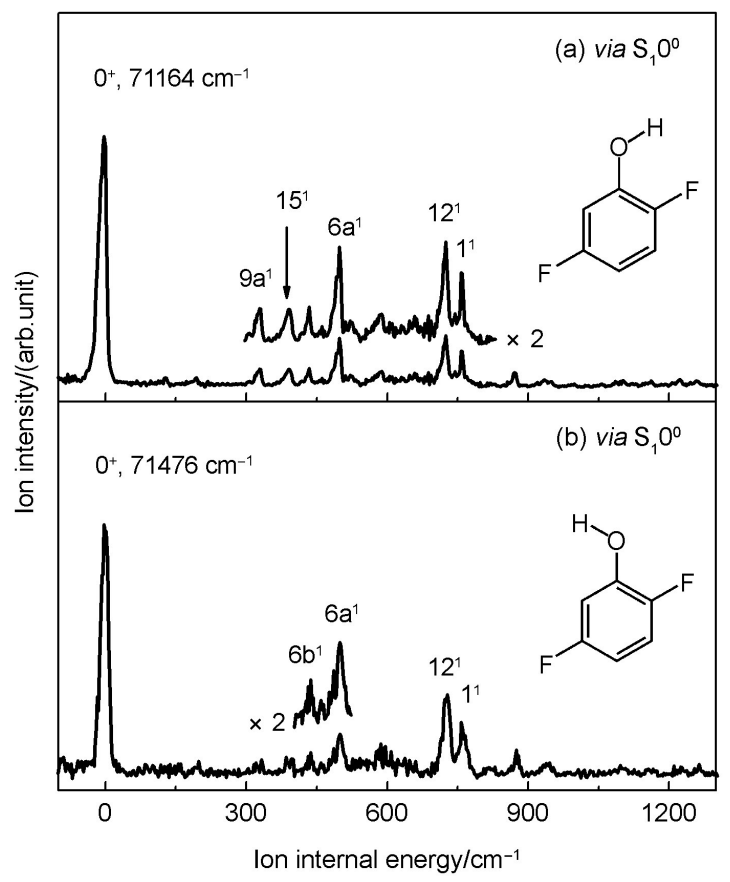

Fig.3 MATI spectra of (a) cis-2,5-difluorophenol and (b) trans2,5-difluorophenol, recorded by ionizing via their respective $S_{1} 0^{0}$ states at 36448 and $36743 \mathrm{~cm}^{-1}$
Table 2 Observed bands (in $\mathrm{cm}^{-1}$ ) in the MATI spectra of cis-2,5-difluorophenol ${ }^{\mathrm{a}}$

\begin{tabular}{|c|c|c|c|c|c|c|}
\hline \multirow[b]{2}{*}{$0^{\circ}$} & \multirow[b]{2}{*}{$10 b^{1}$} & \multirow[b]{2}{*}{$6 a^{1}$} & \multicolumn{3}{|c|}{ Intermediate level in the $S_{1}$ state } & \multirow{2}{*}{$\begin{array}{c}\text { Assignment and } \\
\text { approximate description }\end{array}$} \\
\hline & & & $12^{1}$ & $1^{1}$ & Cal. shift ${ }^{a}$ & \\
\hline & 260 & & & & & $10 \mathrm{~b}^{2}, \gamma(\mathrm{C}-\mathrm{OH}), \gamma(\mathrm{C}-\mathrm{F})$ \\
\hline 331 & 331 & 331 & & & 308 & $9 \mathrm{a}^{1}, \beta(\mathrm{C}-\mathrm{OH}), \beta(\mathrm{C}-\mathrm{F})$ \\
\hline 389 & 388 & 386 & & & 354 & $15^{1}, \beta(\mathrm{C}-\mathrm{F})$ \\
\hline 434 & & & & & 400 & $6 b^{1}, \beta(C C C)$ \\
\hline \multirow[t]{3}{*}{501} & & 501 & & & 510 & $6 a^{1}, \beta(C C C)$ \\
\hline & 519 & & & & & $10 b^{4}$ \\
\hline & & 659 & & & & $9 a^{2}$ \\
\hline 725 & & & 729 & 730 & 734 & $12^{1}, \beta(\mathrm{CCC})$ \\
\hline 758 & & & 763 & 766 & 771 & $1^{1}$, breathing \\
\hline \multirow[t]{6}{*}{866} & & & & & & $6 b^{2}$ \\
\hline & & & & 933 & & $6 \mathrm{a}^{1} 6 \mathrm{~b}^{1}$ \\
\hline & & & 982 & & & $12^{1} 10 b^{2}$ \\
\hline & & 999 & & & & $6 a^{2}$ \\
\hline & & & & 1041 & & $12^{1} 9 \mathrm{a}^{1}$ \\
\hline & & & & 1149 & & $1^{1} 15^{1}$ \\
\hline
\end{tabular}

${ }^{\mathrm{a}}$ The experimental values are shifts from $71164 \mathrm{~cm}^{-1}$, whereas the calculated ones are obtained from the UB3LYP/6-311++ $\mathrm{G}(d, p)$ calculations

(without being scaled). ${ }^{\mathrm{b}} \beta$, in-plane bending; $\gamma$, out-of-plane bending.

most of the resulting active cation vibrations likely involve substituent-sensitive motions. The bands at 260, 331, 388, and 519 are tentatively assigned to the $10 \mathrm{~b}^{2}, 9 \mathrm{a}^{1}, 15^{1}$, and $10 \mathrm{~b}^{4}$ vibrations, respectively. In addition to these substituent-sensitive modes, many in-plane ring deformation vibrations of the cis-2,5-difluorophenol cation are observed. For example, vibrations 6b, 6a, 12, and 1 are found to have frequencies of 434, 501, 729, and 766 $\mathrm{cm}^{-1}$, respectively.

As shown in Fig.3(b) and Table 3, the active vibrations of the trans-2,5-difluorophenol cation include $6 \mathrm{~b}^{1}, 6 \mathrm{a}^{1}, 12^{1}$, and $1^{1}$ with frequencies of $434,501,725$, and $758 \mathrm{~cm}^{-1}$, respectively. For each of these in-plane ring deformation modes, the vibrational frequency of trans-2,5-difluorophenol is very close to that of cis2,5-difluorophenol. This result indicates that relative configuration of the $\mathrm{OH}$ substituent with respect to the $\mathrm{F}$ atom has little effect on these normal vibrations of the 2,5-difluorophenol cation. In other words, the weak intramolecular $\mathrm{OH} \cdots \mathrm{F}$ through-space interaction has little influence on the frequency of the in-plane ring vibration.

\section{Discussion}

\subsection{Energy level diagram and $\mathrm{OH} \cdots \mathrm{F}$ through-space interaction}

Previous spectroscopic studies show that only the cis form involves in the photo-excitation and ionization processes of 2fluorophenol ${ }^{14,15}$ and 2,4-difluorophenol ${ }^{11,12}$. In the cases of 3-fluorophenol $^{18}$ and 3,4-difluorophenol ${ }^{13}$, both cis and trans rotamers have been detected in the $S_{1}$ and $D_{0}$ states. The weak through-space intramolecular interaction between the $\mathrm{F}$ and $\mathrm{OH}$ substituents may be accountable for these experimental results. It is intuitively for one to expect a similar through-space $\mathrm{OH} \cdots \mathrm{F}$ substituent-sub- 


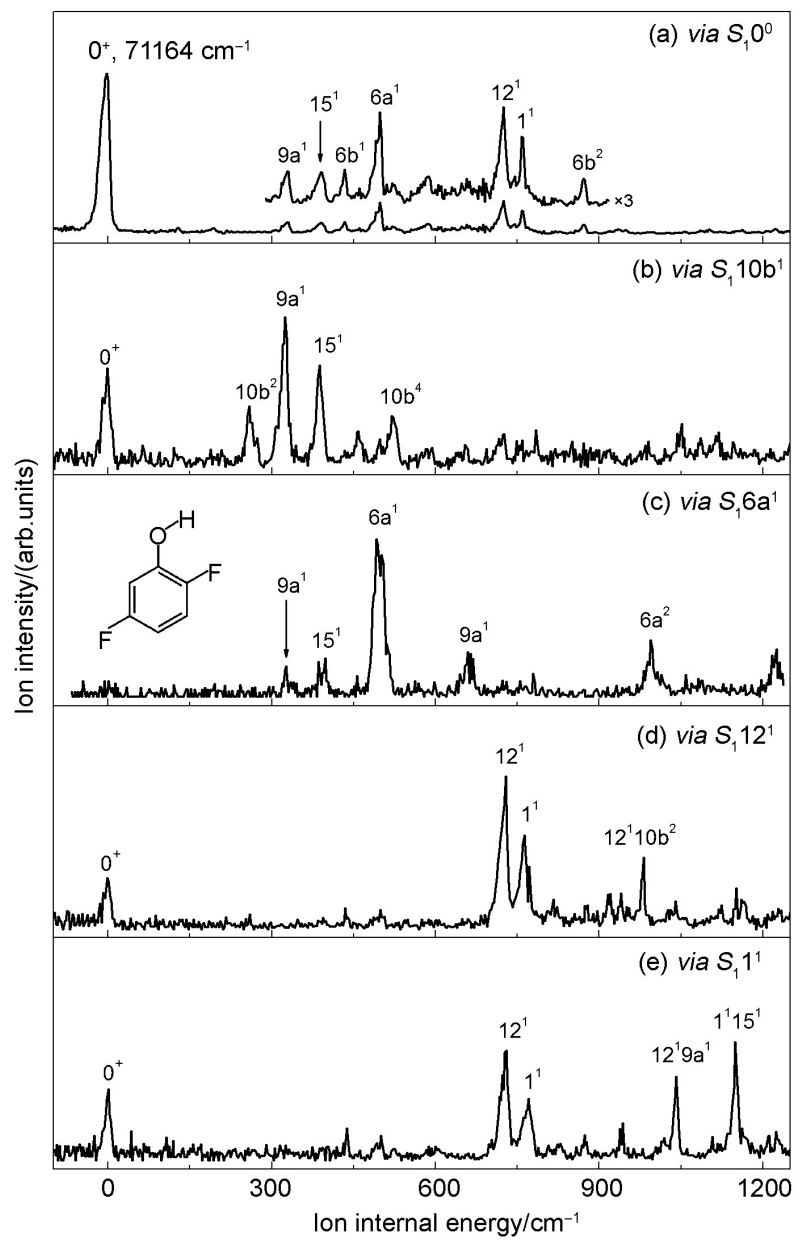

Fig.4 MATI spectra of cis-2,5-difluorophenol recorded by ionizing via its (a) $0^{0}$, (b) $10 \mathrm{~b}^{1}$, (c) $6 \mathrm{a}^{1}$, (d) $12^{1}$, and

(e) $1^{1}$ levels in the $S_{1}$ state

Table 3 Observed bands $\left(\right.$ in $\mathrm{cm}^{-1}$ ) in the MATI spectra of trans-2,5-difluorophenol ${ }^{\mathrm{a}}$

\begin{tabular}{ccc}
\hline \multicolumn{2}{c}{ Intermediate level in the $S_{1}$ state } & $\begin{array}{c}\text { Assignment and } \\
\text { approximate description }^{\mathrm{b}}\end{array}$ \\
\hline $0^{0}$ & Cal. shift $^{\mathrm{a}}$ & $6 \mathrm{~b}^{1}, \beta(\mathrm{CCC})$ \\
534 & 407 & $6 \mathrm{a}^{1}, \beta(\mathrm{CCC})$ \\
701 & 508 & $12^{1}, \beta(\mathrm{CCC})$ \\
758 & 737 & $1^{1}$, breathing \\
\hline
\end{tabular}

${ }^{\mathrm{a}}$ The experimental values are shifts from $71476 \mathrm{~cm}^{-1}$, whereas the calculated ones are obtained from the UB3LYP/6-311++G $(d, p)$ calculations

(without being scaled). ${ }^{\mathrm{b}} \beta$, in-plane bending; $\gamma$, out-of-plane bending.

stituent interaction to exist in 2,5-difluorophenol.

Our B3LYP/6-311++G $(d, p)$ calculations show that in the $S_{0}$ state the ZPE of cis-2,5-difluorophenol is lower than that of trans2,4-difluorophenol by $1012 \mathrm{~cm}^{-1}$. As stated previously, the $E_{1}$ of cis- and trans-2,5-difluorophenol are measured to be 36448 and $36743 \mathrm{~cm}^{-1}$, respectively. These experimental and calculated results suggest that the ZPE in the $S_{1}$ state of the cis rotamer is lower than that of the trans rotamer by $1307 \mathrm{~cm}^{-1}$, as shown in Fig.5. This implies that the cis rotamer has a slightly stronger $\mathrm{OH} \cdots \mathrm{F}$ through-space interaction than the trans rotamer in the $S_{1}$ state as that in the $S_{0}$ state. In a similar fashion, Fujii and coworkers ${ }^{41}$ proposed an enhanced interaction of the $\mathrm{NH}_{2}$ and $\mathrm{OH}$ substituents for the cis rotamer in their spectroscopic study of 3aminophenol. With the measured IE and calculated values, one estimates that the cis rotamer lies in a lower energy level than that of the trans rotamer by $1324 \mathrm{~cm}^{-1}$, as shown in Fig.5. These results suggest that in all $S_{0}, S_{1}$, and $D_{0}$ states cis-2,5-difluorophenol has an $\mathrm{OH} \cdots \mathrm{F}$ through-space interaction, where trans-2,5-difluorophenol does not have. Tsai and Tzeng ${ }^{13}$ reported that in the $S_{0}$ state cis-3,4-difluorophenol lies in a slightly higher energy level than trans-3,4-difluorophenol by $71 \mathrm{~cm}^{-1}$. However, the cis rotamer lies in a slightly lower energy level that the trans rotamer in the $S_{1}$ and $D_{0}$ states by no more than $150 \mathrm{~cm}^{-1}$. Because the two $\mathrm{F}$ atoms are far away from the $\mathrm{OH}$ substituent, the through-space interaction of the $\mathrm{OH}$ and $\mathrm{F}$ substituents of 3,4-difluorophenol is negligible. Therefore, the strength of through-space substituent - substituent interaction depends on the nature and location of the substituents as well as the electronic state of these rotameric species.

\subsection{Transition energy and additivity rule}

Table 4 lists the measured $E_{1}$ and IEs of phenol and its fluorine substituted derivatives on the basis of the LIF, REMPI, ZEKE, and MATI experiments ${ }^{14-18,42}$. The $E_{1}$ of 2-fluorophenol is higher than that of phenol by $451 \mathrm{~cm}^{-1}$, and those of cis- and trans-3-fluorophenol are greater by 274 and $480 \mathrm{~cm}^{-1}$, respectively. The fluorine atom can interact with the aromatic ring through the $\sigma$ bond by the inductive effect and via the $\pi$ orbital overlap. The collective

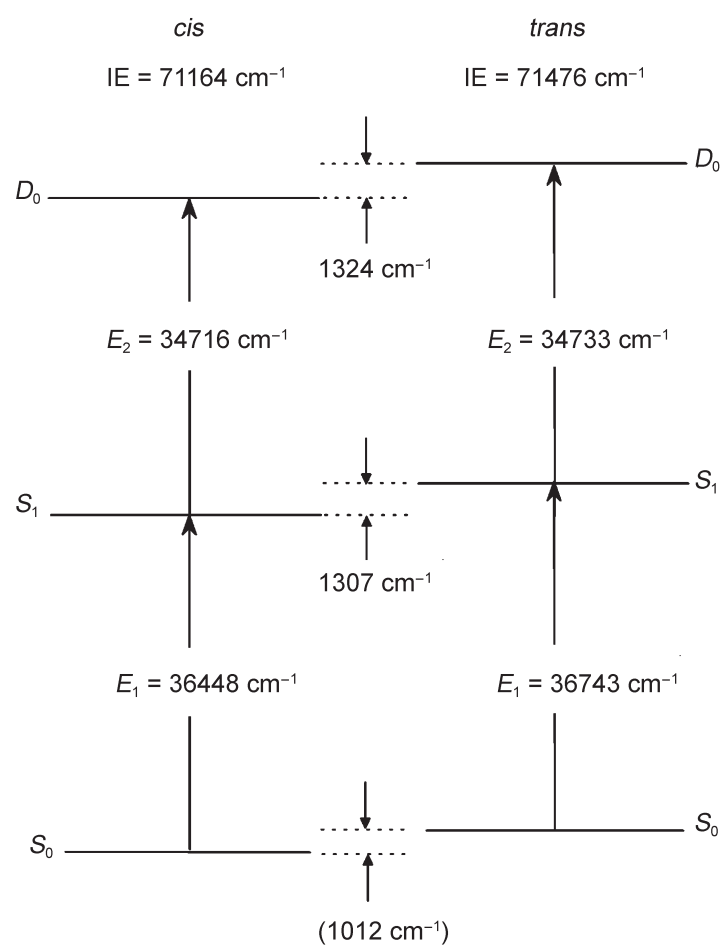

Fig.5 Energy level diagram of the cis and trans rotamers of 2,5-difluorophenol in the $S_{0}, S_{1}$, and $D_{0}$ states

The energy is in unit of $\mathrm{cm}^{-1}$. The relative energy of the two rotamers in the $S_{0}$ state is obtained from the $\mathrm{B} 3 \mathrm{LYP} / 6-311++\mathrm{G}(d, p)$ calculations, whereas those in the $S_{1}$ and $D_{0}$ states are deduced from the measured values in our R2PI and MATI experiments. 
Table 4 Measured transition energies $\left(\mathrm{cm}^{-1}\right)$ of phenol and its fluorine substituted derivatives ${ }^{\mathrm{a}}$

\begin{tabular}{|c|c|c|c|c|c|c|}
\hline Molecule & $E_{1}$ & $\delta E_{1}$ & $E_{2}$ & $\delta E_{2}$ & IE & $\delta \mathrm{IE}$ \\
\hline phenol $^{\mathrm{b}}$ & 36349 & 0 & 32276 & 0 & 68625 & 0 \\
\hline 2-fluorophenol ${ }^{\mathrm{C}}$ & 36800 & 451 & 33206 & 930 & 70006 & 1381 \\
\hline 3-fluorophenol, cis ${ }^{\mathrm{d}}$ & 36623 & 274 & 33565 & 1289 & 70188 & 1563 \\
\hline 3-fluorophenol, $\operatorname{trans}^{\mathrm{d}}$ & 36829 & 480 & 33620 & 1344 & 70449 & 1824 \\
\hline 2,5-difluorophenol, $c i s^{\mathrm{e}}$ & 36448 & 99 & 34716 & 2440 & 71164 & 2539 \\
\hline 2,5-difluorophenol, trans $^{\mathrm{e}}$ & 36743 & 394 & 34733 & 2457 & 71476 & 2851 \\
\hline
\end{tabular}

${ }^{\mathrm{a}} \delta E_{1}, \delta E_{2}$, and $\delta \mathrm{IE}$ are energy shifts of $E_{1}, E_{2}$, and IE with respect to those of phenol. ${ }^{\mathrm{b}} \operatorname{Ref} .42,{ }^{\mathrm{c}} \mathrm{Ref} .16,{ }^{\mathrm{d}} \mathrm{Ref} .18$, ${ }^{\mathrm{e}}$ this work

result of this substituent-ring interaction leads to a blue shift in the $E_{1}$ for the F substitution at the ortho and meta positions of phenol, as seen in Table 4.

One may consider whether the additivity rule ${ }^{13,20}$ can be used to estimate the electronic excitation energy of 2,5-difluorophenol before performing the R2PI experiments for recording the vibronic spectrum as follows. The calculated energy shift $\left(\delta E_{1}\right)$ of $274 \mathrm{~cm}^{-1}$ [for cis-3-fluorophenol] and $451 \mathrm{~cm}^{-1}$ [for 2-fluorophenol] is 725 $\mathrm{cm}^{-1}$, which deviates from the experimental $\delta E_{1}$ of $99 \mathrm{~cm}^{-1}$ for cis2,5-difluorophenol by as much as $626 \mathrm{~cm}^{-1}$. Moreover, the $\delta E_{1}$ of trans-2,5-difluorophenol is estimated to be $931 \mathrm{~cm}^{-1}(=480+451$ $\left.\mathrm{cm}^{-1}\right)$. This value differs from the experimental $\delta E_{1}$ of $394 \mathrm{~cm}^{-1}$ by as much as $537 \mathrm{~cm}^{-1}$ for trans-2,5-difluorophenol. This additivity propensity allows us to predict the $E_{1}$ of 2,5-difluorophenol to no more than $4 \mathrm{~nm}$. A previous study ${ }^{13}$ shows that the corresponding values for cis-3,4-difluorophenol and trans-3,4-difluorophenol are 95 and $107 \mathrm{~cm}^{-1}$. The difference between the estimated $E_{1}$ and measured value is small for 3,4-difluorophenol.

The $D_{0} \leftarrow S_{1}$ transition of the present two-photon R2PI and MATI experiments of 2,5-difluorophenol likely corresponds to the removal of one electron from the aromatic system. The electronwithdrawing $\mathrm{F}$ atom causes a decrease in the electron density on the aromatic ring as well as the $\mathrm{OH}$ group. Therefore, the energies of the $D_{0} \leftarrow S_{1}$ transition $\left(E_{2}\right)$ of 2-fluorophenol, cis- and trans-3fluorophenol are greater than that of phenol, as seen in Table 4. Similar to that stated above, the calculated $\delta E_{2}$ of $2219 \mathrm{~cm}^{-1}(=930+$ $1289 \mathrm{~cm}^{-1}$ ) is different from the experimental value of $2440 \mathrm{~cm}^{-1}$ for cis-2,5-difluorophenol by as small as $221 \mathrm{~cm}^{-1}$. Furthermore, the calculated $\delta E_{2}$ is $2274 \mathrm{~cm}^{-1}\left(=930+1344 \mathrm{~cm}^{-1}\right)$ which differs from the experimental $\delta E_{2}$ of $2457 \mathrm{~cm}^{-1}$ by only $183 \mathrm{~cm}^{-1}$ for trans2,5-difluorophenol. This shows that the additivity rule propensity allows us to set the probe laser to monitor the $D_{0} \leftarrow S_{1}$ transition of the cis and trans rotamers of 2,5-difluorophenol to no more than $2 \mathrm{~nm}$.

\subsection{Cis-trans isomerization energy barrier}

Because the density functional theory (DFT) method does not give a convergent result for the $S_{1}$ state, we performed the HartreeFock (HF) calculations to investigate the cis-trans isomerizations of 2,5-difluorophenol in the $S_{0}, S_{1}$, and $D_{0}$ states. These calculations yield the total electronic energy $\left(E_{\text {total }}\right)$ by scanning one-dimensional potential energy surface on the rotation of ring-hydroxyl bond while all other geometric parameters are fixed. The dihedral angle $\theta$ (or $\mathrm{C} 2 \mathrm{C} 1 \mathrm{OH}$ ) defines the ring-hydroxyl bond with respect to the plane of the aromatic ring. Fig.6 shows the one- dimensional potential energy surfaces of 2,5-difluorophenol in the $D_{0}, S_{1}$, and $S_{0}$ states, obtained from the unrestricted HF (UHF), configuration interaction singles (CIS), and restricted HF (RHF) calculations with the $6-311++\mathrm{G}(d, p)$ basis set, respectively. It is noted that the obtained $E_{\text {total }}$ is related to the bottom of potential well rather than the ZPE level. The calculated results indicate that the cis configuration is most stable in all these three states. They predict that the energy barrier for the trans rotamer to become the cis rotamer is $569 \mathrm{~cm}^{-1}$ in the $S_{0}$ state, $1787 \mathrm{~cm}^{-1}$ in the $S_{1}$ state, and $1795 \mathrm{~cm}^{-1}$ in the $D_{0}$ state. Clearly, the energy barriers of the $S_{1}$ and $D_{0}$ states are about three times of that of the $S_{0}$ state. A possible explanation is that the interaction between the hydroxyl group and

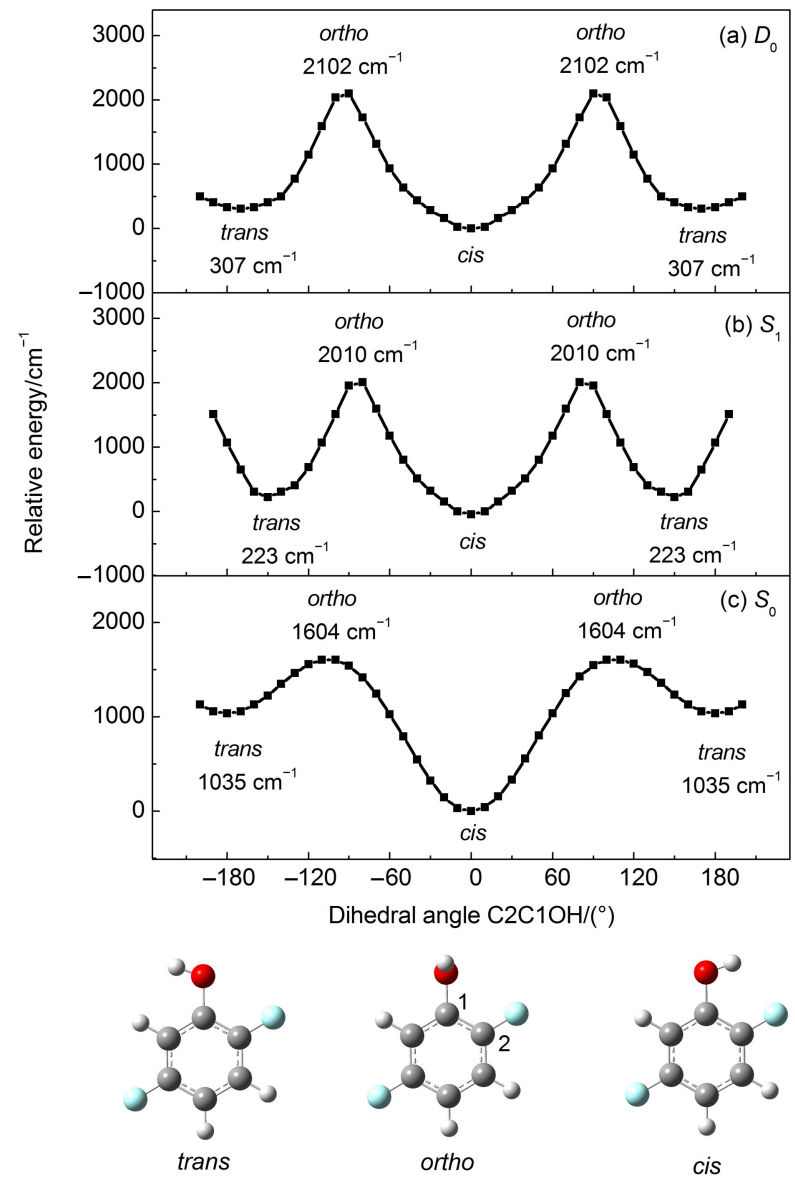

Fig.6 One-dimensional potential energy surfaces of 2,5difluorophenol in the (a) $D_{0}$, (b) $S_{1}$, and (c) $S_{0}$ states, obtained from the UHF, CIS, RHF calculations with the $6-311++\mathrm{G}(d, p)$ basis set 
the ring of 2,5-difluorophenol in the $S_{1}$ or $D_{0}$ state is slightly stronger than that in the $S_{0}$ state. These calculated results support our experimental finding that isomerization of 2,5-difluorophenol does not occur in the UV photoexcitation and ionization processes.

\subsection{Vibrational frequency and molecular geometry}

2,5-Difluorophenol has 33 normal vibrational modes. The $S_{1} \leftarrow$ $S_{0}$ transition is likely subject to a $\pi^{*} \leftarrow \pi$ electronic excitation, leading to a ring expansion and significant change in molecular geometry. Because the intensity of the each vibronic band is related to Franck-Condon overlaps, not all transitions can be observed in the vibronic spectrum. Analysis of the vibronic spectrum in Fig.2 suggests that most of the observed active vibrations in the $S_{1}$ state are related to the in-plane ring deformation and substituentsensitive bending modes. In the two-color resonant two-photon MATI experiments, the intensity of the observed MATI band is related to (1) the oscillator strength of the $S_{1} \leftarrow S_{0}$ transition, (2) the transition cross section from the $S_{1}$ state to the Rydberg state, (3) the pulsed field ionization efficiency, and (4) the overlap integral between the vibrational coordinates of the neutral and the cation. Because of the above reasons, we could only record the MATI spectrum of trans-2,5-difluorophenol, by ionizing via the $S_{1} 0^{0}$ state, as seen in Fig.2(b). However, we were able to record the MATI spectra of cis-2,5-difluorophenol by ionizing via several intermediate vibronic states. When the $S_{1} 6 \mathrm{a}^{1}, S_{1} 12^{1}$, and $S_{1} 1^{1}$ states are used as the intermediate levels, the MATI bands resulting from the same in-plane vibrations of the cis-2,5-difluorophenol cation in the $D_{0}$ state appear to be very strong, as seen in Fig.4(c-e). This observed $\Delta v=0$ propensity implies a good Franck-Condon correspondence associated with the $D_{0} \leftarrow S_{1}$ transition. It indicates that the geometry and vibrational patterns of these modes of cis2,5-difluorophenol are alike for both the $S_{1}$ and $D_{0}$ states. Similar observations have been reported for cis-3-fluorostyrene $^{43}, 3$-fluoroanisole $^{44}, 3,4$-difluoroanisole ${ }^{45}$, and 3,4-difluorophenol ${ }^{13}$.

In-plane ring deformation vibrations $6 \mathrm{a}, 12$, and 1 of $\mathrm{cis}-2,5$ difluorophenol are measured to have respective frequencies of 439,705 , and $718 \mathrm{~cm}^{-1}$ in the neutral $S_{1}$ state and 501, 725, and $758 \mathrm{~cm}^{-1}$ in the cationic $D_{0}$ state. Similarly, modes $6 \mathrm{~b}$, and $6 \mathrm{a}$ of trans-2,5-difluorophenol have respective frequencies of 396 and $446 \mathrm{~cm}^{-1}$ in the $S_{1}$ state and 434 and $501 \mathrm{~cm}^{-1}$ in the $D_{0}$ state. These experimental data show that frequencies of in-plane ring deformation vibrations in the $D_{0}$ state are slightly greater than those in the $S_{1}$ state for these two rotamers of 2,5-difluorophenol. It is known that frequency is proportional to the square root of force constant. The observed higher frequency of the ring deformation vibrations implies that the molecular geometry of 2,5-difluorophenol in the $D_{0}$ state is slightly tighter than that in the $S_{1}$ state.

\section{Conclusions}

We applied the R2PI and MATI techniques to record the vibronic and cation spectra of cis- and trans-2,5-difluorophenol in the $S_{1}$ and $D_{0}$ states. The $E_{1}$ values of the cis and trans rotamers are found to be 36448 and $36743 \mathrm{~cm}^{-1}$, whereas the adiabatic IEs are determined to be 71164 and $71476 \mathrm{~cm}^{-1}$, respectively. Our B3LYP/
$6-311++\mathrm{G}(d, p)$ calculations predict that in the $S_{0}$ state the ZPE of cis-2,5-difluorophenol is lower than that of trans-2,5-difluorophenol by $1012 \mathrm{~cm}^{-1}$. Therefore, the ZPEs in the $S_{1}$ and $D_{0}$ states of the cis rotamer are deduced to be lower than those of the trans by 1307 and $1324 \mathrm{~cm}^{-1}$, respectively. This implies that the cis rotamer in the $S_{0}, S_{1}$, and $D_{0}$ states may exhibit a intramolecular $\mathrm{OH} \cdots \mathrm{F}$ interaction.

With the available spectroscopic data of phenol, 2-fluorophenol, and cis- and trans-3-fluorophenol, we applied the additivity propensity to locate the band origin of the $S_{1} \leftarrow S_{0}$ electronic transition and ionization threshold. This allows us to set the wavelengths of the pump and probe lasers for our R2PI and MATI experiments to record the vibronic and cation spectra of 2,5difluorophenol in a very convenient way.

The observed active modes of cis- and trans-2,5-difluorophenol in the $S_{1}$ and $D_{0}$ states include the in-plane ring deformation and substituent-sensitive bending vibrations. Analysis of the MATI spectra recorded by ionizing via several intermediate levels shows that a propensity of maintaining the same vibration in the $D_{0} \leftarrow$ $S_{1}$ transition exists. This indicates that the molecular geometry and vibrational coordinates of the cation in the $D_{0}$ state resemble those of the neutral species in the $S_{1}$ state for cis-2,5-difluorophenol. In addition, investigations on the frequencies of in-plane ring deformation vibrations $6 b^{1}, 6 a^{1}, 12^{1}$, and $1^{1}$ suggest that the geometries of cis- and trans-2,5-difluorophenol are more rigid in the cationic $D_{0}$ state than those in the neutral $S_{1}$ state.

\section{References}

(1) Ribero da Silva, M. A. V.; Lobo Ferreira, A. I. M. C. J. Chem. Thermodyn. 2010, 42, 133. doi: 10.1016/j.jct.2009.07.021

(2) Rao, R.; Aralakkanavar, M. K.; Rao, K. S.; Shashidhar, M. A. Spectrochim. Acta A 1989, 45, 103. doi: 10.1016/0584-8539 (89)80110-2

(3) Kolek, P.; Pirowska, K.; Gora, M.; Kozik, B.; Najbar, J. Chem. Phys. 2002, 285, 55. doi: 10.1016/S0301-0104(02) 00689-4

(4) Kolek, P.; Pirowska, K.; Chacaga, L.; Najbar, J. Phys. Chem. Chem. Phys. 2003, 5, 4096. doi: 10.1039/B305797F

(5) Itoh, T.; Tanaka, N.; Tsukada, Y.; Nishikiori, H.; Fujii, T. J. Mol. Struct. 2011, 1000, 35. doi: 10.1016/j. molstruc.2011.05.046

(6) Yoon, Y. K.; Huh, C. S.; Lee, S. K. Chem. Phys. Lett. 2012, 550, 58. doi: 10.1016/j.cplett.2012.08.060

(7) Huh, C. S.; Yoon, Y. W.; Lee, S. K. J. Chem. Phys. 2012, 136, 174306. doi: 10.1063/1.4708808

(8) Ito, M.; Oikawa, A. J. Mol. Struct. 1985, 126, 133. doi: 10.1016/0022-2860(85) 80109-5

(9) Kazmierski, W. M.; Danehower, S.; Duan, M.; Ferris, R. G.; Elitzin, V.; Minick, D.; Sharp, M.; Stewart, E.; Villeneuve, M.; Med. Chem. Lett. 2014, 5, 1296. doi: 10.1021/m15004124

(10) Ortega, D. R.; Yang, C.; Ames, P.; Baudry, J.; Parkinson, J. S.; Zhulin, I. B. Nat. Commun. 2013, 4, 1. doi: 1038/ncomms3881

(11) Chakrabarti, S.; Jaman, A. I. J. Mol. Struct. 2002, 642, 93. doi: 
10.1016/S0022-2860(02) 00398-8

(12) Shivatare, V.; Tzeng, W. B. Bull. Korean Chem. Soc. 2014, 35, 815. doi: $10.5012 / \mathrm{bkcs} .2014 .35 .3 .815$

(13) Tsai, C. Y.; Tzeng, W. B. J. Photochem. Photobiol. A 2013, 270, 53. doi: 10.1016/j.jphotochem.2013.07.014

(14) Fujii, A.; Iwasaki, A.; Mikami, N. Chem. Lett. 1997, 11, 1099. doi: 10.1246/cl.1997.1099

(15) Fujimaki, E.; Fujii, A.; Ebata, T.; Mikami, N. J. Chem. Phys. 1999, 110, 4238. doi: 10.1063/1.478306

(16) Yuan, L.; Li, C.; Lin, J. L.; Yang, S. C.; Tzeng, W. B. Chem. Phys. 2006, 323, 429. doi: 10.1016/j.chemphys.2005.10.004

(17) Oikawa, A.; Abe, H.; Mikami, N.; Ito, M. Chem. Phys. Lett. 1985, 116, 50. doi: 10.1016/0009-2614(85) 80123-8

(18) Yosida, K.; Suzuki, K.; Ishiuchi, S.; Sakai, M.; Fujii, M.; Dessent, C. E. H.; Muller-Dethlefs, K. Phys. Chem. Chem. Phys. 2002, 4, 2534. doi: 10.1039/B201107G

(19) Zhang, B.; Li, C.; Su, H.; Lin, J. L.; Tzeng, W. B. Chem. Phys. Lett. 2004, 390, 65. doi: 10.1016/j.cplett.2004.04.013

(20) Huang, W. C.; Huang, P. S.; Hu, C. H.; Tzeng, W. B. Chem. Phys. Lett. 2013, 580, 28. doi: 10.1016/j.cplett.2013.06.053

(21) Ribeiro-Claro, P. J. A.; Teixeira-Dias, J. J. C.; Hollas, J. M.; Milewski, M. J. Chem. Soc. Faraday Trans. 1995, 91, 197. doi: 10.1039/FT9959100197

(22) Breen, P. J.; Bernstein, E. R.; Secor, H. V.; Seeman, J. I. J. Am. Chem. Soc. 1989, 111, 1958. doi: 10.1021/ja00188a002

(23) Li, C.; Lin, J. L.; Tzeng, W. B. J. Chem. Phys. 2005, 122, 044311. doi: 10.1063/1.1839863

(24) Gerhards, M.; Perl, W.; Kleinermanns, K. Chem. Phys. Lett. 1995, 240, 506. doi: 10.1016/0009-2614(95) 00567-N

(25) Patwari, G. N.; Doraiswamy, S.; Wategaonkar, S. Chem. Phys. Lett. 1998, 289, 8. doi: 10.1016/S0009-2614(98) 00398-4

(26) Telle, H. H.; Urena, A. G.; Donovan, R. J. Laser Chemistry; John Wiley \& Sons, Ltd.: West Sussex, England, 2007.

(27) Müller-Dethlefs, K.; Sander, K. M.; Schlag, E. W. Chem. Phys. Lett. 1984, 112, 291. doi: 10.1016/0009-2614(84) 85743-7

(28) Müller-Dethlefs, K.; Dopfer, O.; Wright, T. G. Chem. Rev. 1994, 94, 1845. doi: 10.1021/cr00031a006
(29) Cockett, M. C. R. Chem. Soc. Rev. 2005, 34, 935. doi: 10.1039/ b505794a

(30) Zhu, L.; Johnson, P. J. Chem. Phys. 1991, 94, 5769. doi: $10.1063 / 1.460460$

(31) Dessent, C. E. H.; Haines, S.; Müller-Dethlefs, K. Chem. Phys. Lett. 1999, 315, 103. doi: 10.1016/S0009-2614(99) 01193-8

(32) Georgiev, S.; Karaminkov, R.; Chervenkov, S.; Delchev, V.; Neusser, H. J. J. Phys. Chem. A 2009, 113, 12328. doi: $10.1021 / \mathrm{jp} 908045 \mathrm{k}$

(33) Witte, F.; Riese, M.; Gunzer, F.; Grotemeyer, J. Int. J. Mass Spectrom. 2011, 306, 129. doi: 10.1016/j.ijms.2010.10.002

(34) Lin, J. L.; Tzeng, W. B. Appl. Spectrosc. 2003, 57, 1178. doi: 10.1366/00037020360696062

(35) Xu, Y.; Tzeng, S. Y.; Shivatare, V.; Takahashi, K.; Zhang, B.; Tzeng, W. B. J. Chem. Phys. 2015, 142, 124314. doi: 10.1063/ 1.4916052

(36) Frisch, M. J.; Trucks, G. W.; Schlegel, H. B.; et al. Gaussian 09, Revision A.02; Gaussian Inc.: Wallingford, CT, 2009.

(37) Dennington, R.; Keith, T.; Millam, J.; GaussView, Version 5; Semichem Inc. :Shawnee Mission KS, 2009.

(38) Varsanyi, G.; Szoke, S. Vibrational Spectra of Benzene Derivatives; Academic Press: London, 1969.

(39) Varsanyi, G. Assignments of Vibrational Spectra of Seven Hundred Benzene Derivatives; Wiley: New York, 1974.

(40) Wilson, E. B. Phys. Rev. 1934, 45, 406. doi: 10.1103/ PhysRev.45.706

(41) Shinozaki, M.; Sakai, M.; Yamaguchi, S.; Fujioka, T.; Fujii, M. Phys. Chem. Chem. Phys. 2003, 5, 5044. doi: 10.1039/ B309461H

(42) Dopfer, O.; Müller-Dethlefs, K. J. Chem. Phys. 1994, 101, 8508. doi: $10.1063 / 1.468111$

(43) Wu, P. Y.; Tzeng, W. B. J. Mol. Spectrosc. 2015, 316, 72. doi: 10.1016/j.jms.2015.06.011

(44) Shiung, K. S.; Yu, D.; Huang, H. C.; Tzeng, W. B. J. Mol. Spectrosc. 2012, 274, 43. doi: 10.1016/j.jms.2012.04.004

(45) Xu, Y.; Tzeng, S. Y.; Zhang, B.; Tzeng, W. B. Spectrochim. Acta A 2013, 102, 365. doi: 10.1016/j.saa.2012.10.020 